\title{
3D color charts for camera spectral sensitivity estimation
}

\author{
Rada Deeb ${ }^{1}$ \\ Rada.deeb@gmail.com \\ Damien Muselet ${ }^{1}$ \\ damien.muselet@univ-st-etienne.fr \\ Mathieu Hebert ${ }^{1}$ \\ mathieu.hebert@institutoptique.fr \\ Alain Tremeau ${ }^{1}$ \\ alain.tremeau@univ-st-etienne.fr \\ Joost van de Weijer ${ }^{2}$ \\ joost@cvc.uab.es
}

${ }^{1}$ Univ Lyon, UJM-Saint-Etienne, CNRS, LaHC UMR 5516, Institut Optique Graduate School, F-42023, SAINTETIENNE, France

${ }^{2}$ Computer Vision Center - UAB Barcelona - Spain

\begin{abstract}
Estimating spectral data such as camera sensor responses or illuminant spectral power distribution from raw RGB camera outputs is crucial in many computer vision applications. Usually, 2D color charts with various patches of known spectral reflectance are used as reference for such purpose. Deducing n-D spectral data $(n » 3)$ from $3 D$ RGB inputs is an ill-posed problem that requires a high number of inputs. Unfortunately, most of the natural color surfaces have spectral reflectances that are well described by low-dimensional linear models, i.e. each spectral reflectance can be approximated by a weighted sum of the others. It has been shown that adding patches to color charts does not help in practice, because the information they add is redundant with the information provided by the first set of patches. In this paper, we propose to use spectral data of higher dimensionality by using $3 \mathrm{D}$ color charts that create inter-reflections between the surfaces. These inter-reflections produce multiplications between natural spectral curves and so provide non-linear spectral curves. We show that such data provide enough information for accurate spectral data estimation.
\end{abstract}

\section{Introduction}

The outputs of color cameras are the inputs of most of the computer vision algorithms, from which decisions are taken about the observed scene (segmentation [四], tracking [ㅁ] , classification [], object detection [ष] , ...). However, these RGB coordinates do not only characterize intrinsic properties of the observed surfaces since they depend on the light illuminating the scene as well as on the camera characteristics such as the sensor responses. Indeed, the same objects under two different lights or acquired by two different cameras can be characterized by significantly different RGB coordinates [四, $\square, \square]$. Thus, the light spectral power distribution (SPD) and the camera sensor sensitivities are relevant information that can be added as input to computer vision algorithms along with the RGB data. This 
can be particularly important regarding the current trend in computer vision that consists in providing large amount of raw data to deep networks and let them extract and combine the most relevant ones. Furthermore, this knowledge (about the sensor sensitivities and the light SPD) is also essential in many applications such as multispectral imaging, spectral reflectance estimation, color constancy, intrinsic image decomposition from RGB coordinates, shadow removal, etc [ $[\mathbf{\square}, \mathbf{B}, \square, \square]$.

There exist calibrated color charts used to get such spectral data from raw RGB coordinates. As described later in this paper, the idea consists in exploiting a model accounting all the physical elements: the sensor sensitivities, the illuminant SPD, the spectral reflectances of the surfaces constituting the color charts and the RGB coordinates of the pixels. Then, the equation provided by the model is solved for one unknown spectral curve (sensor response or illuminant SPD), while the other parameters are known [G]. In order to get accurate spectral estimation results, one possible approach is to increase the number of $2 \mathrm{D}$ color patches on the observed color chart. Nevertheless, it is known that natural (printed) spectral reflectances are well described by a low-dimensional linear model, i.e. the spectral reflectance of a surface can be well approximated by a weighted sum of other spectral reflectances [ $[\mathbf{D}]$. Consequently, adding new patches to color charts does not help for such kind of applications, as the added spectral reflectances are redundant with the ones already available. This point is well discussed in Darrodi's et al. work [ $[$ ] $]$, where the authors showed that the spectral sensor sensitivities are not better estimated with 1995 surfaces than with only 24 surfaces.

In this paper, we propose to 'simulate' surfaces that would have spectral reflectances not redundant with the classically used ones. This is done by folding homogeneous surfaces and so creating inter-reflections between both sides of these folded surfaces (see Figure 1). The resulting colors are also modeled by a physic-based equation in which the used spectral reflectances are the results of multiplications between the classical spectral reflectances. Consequently, these resulting reflectances can not be so easily approximated by a linear combination of the classical natural reflectances. So, it is not surprising that taking into account such 'non-linear' reflectances in the matrix equation increases the accuracy of the resulted spectral estimation. This would confirm the results of a recent psycho-visual study showing that human observers are better at color constancy (estimating the intrinsic color of a surface under unknown light source) when inter-reflections are present in the scene [ $\mathbb{}$ ].

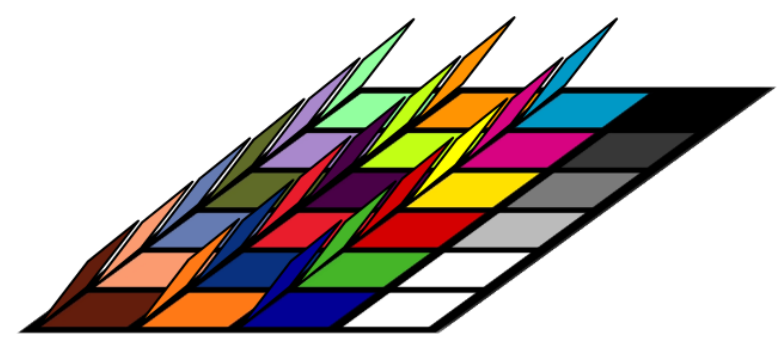

Figure 1: A 3D color chart with inter-reflections.

The idea is illustrated in Figure 2, where we have displayed one homogeneous color patch sampled with $8 \times 14$ facets. When the patch is flat and the illumination is diffuse and far enough from the patch, all the facets have the same colors. When the same patch is folded, creating a concave surface as shown in the top-center illustration of this figure, the facets have different colors due to the inter-reflection effect, that can be described by the 


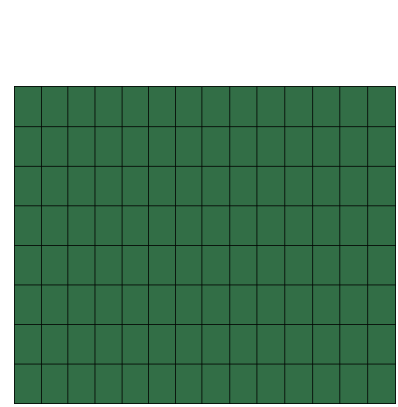

Planar Surface

Constant reflectance

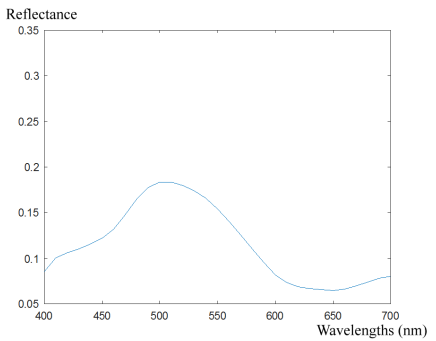

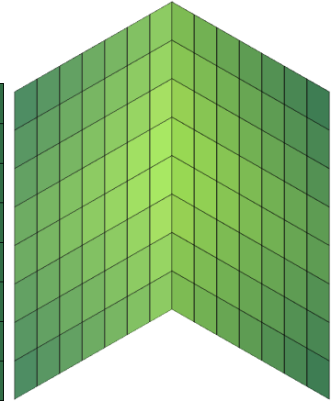

Folded surface

Constant reflectance

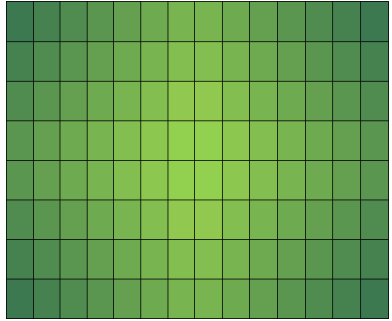

Planar surface

Apparent reflectances

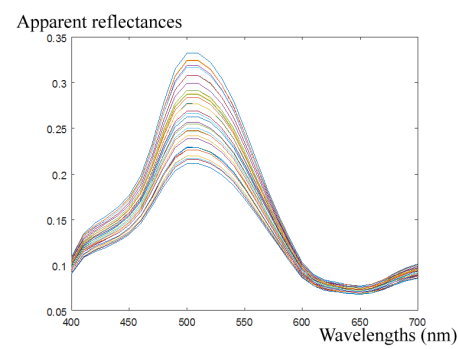

Figure 2: Planar and folded surfaces subdivided into 112 facets. Top-left: one green patch extracted from the Macbeth Color Checker. Bottom-left: the spectral reflectance of this patch. Top-center: The appearance of this patch when folded. We can see how the facet color varies from the central position to the edges. These colors have been evaluated from the inter-reflection model of eq.(8). Top-right: One 'virtual' flat paper whose facets have the same appearance colors as the ones in the folded green paper. Bottom-right: the apparent reflectances of these virtual facets. The equation of these reflectances $\mathbf{R}_{\lambda}^{\text {ap }}$ is detailed later in this paper. We can note that they are more narrow-banded than the natural green reflectance because they are the results of reflectance multiplications.

model exposed later. In Section 3 we show how to estimate the so called apparent spectral reflectances of $2 \mathrm{D}$ facets so that they have the same color as in a folded surface but with only one non-folded surface, as shown in the top-right illustration of this figure. It is worth mentioning that we do not need to measure their reflectances, we just estimate them from our model. The apparent reflectances of these facets are displayed on the bottom-right plot and can be compared with the real reflectance on the bottom-left plot. Because they are obtained by multiplication of the same initial reflectance, they are much more narrow-banded than the natural reflectances and help to extract complementary information in addition to the one extracted from natural reflectances.

In the following, we present the background for spectral data estimation as well as the related works. We concentrate in this paper on the problem of spectral camera response estimation, especially because of the higher dimensionality of this problem compared to illuminant SPD estimation. Off course, the same approach can be applied to illuminant SPD estimation from RGB data. In Section 3, we exploit the results of Nayar et al. [四] to deduce a physical model of inter-reflections. The properties of the obtained inter-reflection apparent 
reflectances are discussed in Section 4 and their exploitation in sensor response estimation is presented in Section 5.

\section{Background and Related works}

In this section, we first present the classical approach used to estimate spectral curves from RGB data and second we explain how inter-reflections have been used to help solving problems in computer vision.

\subsection{Spectral data estimation}

The raw (linear) RGB outputs of a camera, observing a Lambertian surface $S_{i}$ with spectral reflectance $R_{i}(\lambda)$, under an illuminant with $\operatorname{SPD} E(\lambda)$ is given by:

$$
\rho_{i}^{k}=\frac{1}{\pi} \int_{\omega} C^{k}(\lambda) E(\lambda) R_{i}(\lambda) d \lambda,
$$

where $k \in\{R, G, B\}, \omega$ is the spectral range over which the camera is sensitive and $C^{k}(\lambda)$ is the spectral response of the sensor $k$ of the camera.

A discrete version of this equation has the form:

$$
\rho_{\mathbf{i}}=\frac{1}{\pi} \mathbf{C ~ E ~} \mathbf{R}_{\mathbf{i}}
$$

where $\rho_{\mathbf{i}}$ is a $3 \times 1$ vector containing the $3 \mathrm{red}$, green and blue outputs of the pixel, $\mathbf{C}$ is a $3 \times n_{\lambda}$ matrix containing in each row $k$ the sensor sensitivity of the sensor $k$ of the camera, $n_{\lambda}$ being the number of wavebands over the range $\omega, \mathbf{E}$ is a $n_{\lambda} \times n_{\lambda}$ diagonal matrix containing the SPD of the illuminant and $\mathbf{R}_{\mathbf{i}}$ is a $n_{\lambda} \times 1$ vector containing the spectral reflectance of the observed surface $S_{i}$.

In order to estimate the matrix $\mathbf{C}$, more than one pixel with different RGB coordinates are needed. All the $n_{P}$ pixel color coordinates can be stored into a $3 \times n_{P}$ matrix $\rho$ expressed as follows:

$$
\rho=\frac{1}{\pi} \mathbf{C ~ E ~ R , ~}
$$

where $\mathbf{R}$ is a $n_{\lambda} \times n_{P}$ matrix containing, on each column $i$, the spectral reflectance of the observed surface $S_{i}$.

Starting from this equation and considering that we know $\rho, \mathbf{E}$ and $\mathbf{R}$, we can get an estimate $\hat{\mathbf{C}}$ of the sensor responses by using the Moore-Penrose pseudo-inverse:

$$
\hat{\mathbf{C}}=\pi \rho(\mathbf{E ~ R})^{T}\left((\mathbf{E ~ R})(\mathbf{E ~ R})^{T}\right)^{-1} .
$$

This least-square solution is the one that minimizes $\left\|\rho-\frac{1}{\pi} \mathbf{C} \mathbf{E} \mathbf{R}\right\|^{2}$. Regularization terms can be added to this equation in order to prevent high norm sensor sensitivities or to get smoother spectral curves [ [R]].

The number of color patches is crucial to get accurate results because the inputs are 3-D RGB and the outputs are $n_{\lambda}$-D spectral curves $\left(n_{\lambda}>>3\right)$. So the number of independent equations has to be high enough to get accurate solutions. Nevertheless, natural reflectances generally lie in a low dimensional linear space (6-D or 7-D), thus adding new patches introduces no or very little improvement on the reconstruction quality [ $\mathbf{Q}]$. A classical approach 
is to use various illuminants. In this paper, we propose an alternative approach that consists in folding the available natural patches in order to create inter-reflections, which creates variations in color values corresponding to more than one spectral input.

\subsection{Inter-reflections in computer vision}

Inter-reflections occur in concave surfaces or between different nearby surfaces when light beams bounce between surface elements before reaching the eye or the camera sensor in a vision system. The properties of the spectral distribution of the radiances describing the light rays after each bounce depend on the surface spectral reflectance as well as on the geometry of the scene. This phenomenon, that can have a strong impact on the observed colors, has gained a lot of attentions in computer graphics [ $[\square, \square, \mathbb{\square}$, ], but only few methods have been proposed in the literature to handle inter-reflections in computer vision applications. Most of them focus on removing the effect of inter-reflections $[\mathbf{\square}, \boldsymbol{\nabla}, \boldsymbol{\nabla}, \boldsymbol{\square}, \boldsymbol{\square}, \boldsymbol{\nabla}]$, generally for shape from shading applications. On the other side, some other papers proposed to use inter-reflections as additional information to find illumination spectral power distribution and surface spectral reflectance from RGB data [ $\mathbf{Q}, \mathbf{\theta}, \mathbb{\square}]$ ]. For example, Funt et al. [ $[$ ] proposed to use inter-reflections as extra information in order to obtain surface spectral reflectance and illuminant spectral power distribution. However, only one bounce of inter-reflection between surface elements was taken into consideration. In addition, the two surfaces must have different spectral reflectances, and an area on each surface should not be affected by inter-reflection. These conditions are rather hard to meet in real-life situations.

The model that we present hereafter follows a similar aim as Funt et al. [ $[$ ], but it takes into consideration infinite light bounces, which is crucial for good accuracy, and relies on inter-reflections between surfaces having same reflectance. This model will be used in this paper to estimate sensor response from RGB data and could also be used to estimate illuminant SPD.

\section{Inter-reflection model}

Being given a concave surface sampled into $m$ facets $f_{i}$, according to Nayar $e t a l$. []], the radiances for a single wavelength $\lambda$ of the $m$ facets can be described by a vector $\mathbf{L}_{\lambda}$ satisfying the following equation:

$$
\mathbf{L}_{\lambda}=\mathbf{L}_{\lambda}^{\text {no-inter }}+\mathbf{R}_{\lambda} \mathbf{K} \mathbf{L}_{\lambda},
$$

where $\mathbf{L}_{\lambda}^{\mathbf{n o}-\text { inter }}$ is a $m$-dimensional vector containing the respective radiances reflected by the facets in absence of inter-reflections, $\mathbf{R}_{\lambda}$ is a diagonal $m \times m$ matrix containing the spectral reflectances $r_{i, \lambda}$ of each facet $f_{i}, i=1, \ldots, m$ for the considered wavelength $\lambda$. Note that, if the concave surface is homogeneous, with a reflectance $r_{\lambda}$ at wavelength $\lambda$, then $\mathbf{R}_{\lambda}=r_{\lambda} \mathbf{I}_{\mathbf{m}}$, where $\mathbf{I}_{\mathbf{m}}$ is the $m \times m$ identity matrix. It is worth mentioning that this model is considering only Lambertian surfaces. Nevertheless, although the surfaces used in our practical tests are not perfectly Lambertian, we got accurate results. In the previous equation, the matrix $\mathbf{K}$ is called Kernel matrix, whose elements $k_{i j}$ are driven from the geometrical extent subtended by the pair of facets $f_{i}$ and $f_{j}$. This term $k_{i j}$ is expressed as:

$$
k_{i j}=\frac{\left(\vec{N}_{i} \cdot \vec{f}_{i} f_{j}\right)\left(\vec{N}_{j} \cdot \vec{f}_{j} f_{i}\right) V\left(f_{i}, f_{j}\right)}{\Delta_{i j}^{4}},
$$


where $\vec{N}_{i}$ is the normal to the facet $f_{i}$, the point operator (.) is the dot product between two vectors, $\Delta_{i j}$ is the euclidean distance between the centers of the facets $f_{i}$ and $f_{j}$ and $V\left(f_{i}, f_{j}\right)$ is a binary visibility function giving 1 if the two facets can "see" each other and 0 otherwise.

It is worth mentioning that the radiance vector $\mathbf{L}_{\lambda}^{\text {no-inter }}$ that neglects the inter-refections can be expressed with the classical Lambertian reflection equation:

$$
\mathbf{L}_{\lambda}^{\text {no-inter }}=\frac{1}{\pi} \mathbf{R}_{\lambda} \mathbf{E}_{\lambda},
$$

where $\mathbf{E}_{\lambda}$ is a $m$-dimensional vector containing the irradiance, for the considered wavelength $\lambda$, received from the light source by the $m$ facets. Note that if the source is diffuse and far enough from the surface, the elements of this irradiance vector are all the same since all the facets receive the same irradiance from the light source.

From equations (5) and (7), we can deduce the analytical expression of the radiance vector $\mathbf{L}_{\lambda}$ as follows:

$$
\begin{aligned}
\mathbf{L}_{\lambda} & =\frac{1}{\pi} \mathbf{R}_{\lambda} \mathbf{E}_{\lambda}+\mathbf{R}_{\lambda} \mathbf{K} \mathbf{L}_{\lambda}, \\
\mathbf{L}_{\lambda}\left(\mathbf{I}_{\mathbf{m}}-\mathbf{R}_{\lambda} \mathbf{K}\right) & =\frac{1}{\pi} \mathbf{R}_{\lambda} \mathbf{E}_{\lambda}, \\
\mathbf{L}_{\lambda} & =\frac{1}{\pi}\left(\mathbf{I}_{\mathbf{m}}-\mathbf{R}_{\lambda} \mathbf{K}\right)^{-1} \mathbf{R}_{\lambda} \mathbf{E}_{\lambda}, \\
\mathbf{L}_{\lambda} & =\frac{1}{\pi}\left(\mathbf{R}_{\lambda}^{-1}-\mathbf{K}\right)^{-1} \mathbf{E}_{\lambda} .
\end{aligned}
$$

We propose to introduce the matrix $\mathbf{R}_{\lambda}^{\text {ap }}=\left(\mathbf{R}_{\lambda}{ }^{-1}-\mathbf{K}\right)^{-1}$, called hereafter the apparent reflectance matrix. Thus, the previous equation becomes:

$$
\mathbf{L}_{\lambda}=\frac{1}{\pi} \mathbf{R}_{\lambda}^{\mathbf{a p}} \mathbf{E}_{\lambda}
$$

If the kernel matrix is a zero matrix $\mathbf{0}$, i.e. no inter-reflections occur, then $\mathbf{R}_{\lambda}^{\mathrm{ap}}=\left(\mathbf{R}_{\lambda}{ }^{-1}\right.$ $\mathbf{0})^{-1}=\mathbf{R}_{\lambda}$. Hence, inter-reflections modify the reflectance matrix $\mathbf{R}_{\lambda}$ into $\mathbf{R}_{\lambda}^{\mathbf{a p}}$ and change accordingly the output radiance. This means that, if we could have created a flat surface (without inter-reflections) made of facets having the apparent spectral reflectances provided by the matrix $\mathbf{R}_{\lambda}^{\text {ap }}$, we would have obtained the same output radiance vector as if we had observe the considered homogeneous concave surface with inter-reflections. This is why we propose to call this matrix $\mathbf{R}_{\lambda}^{\mathrm{ap}}$ the apparent spectral reflectance matrix. It is important to note that the reflectances in this matrix are calculated with the previous equations and do not need to be measured. In the next sections, the properties of such apparent reflectances are studied in order to measure their impact on the considered application, i.e. sensor response estimation.

\section{Non-linearity of inter-reflections}

It is known that most natural reflectances lie in a low dimension linear space [ $[\mathbf{Q}, \boldsymbol{Q}]$. This is a problem when we want to construct a color chart for camera calibration and spectral reconstruction. Indeed, it has been demonstrated in [G] that adding color patches to the 24 patches of the Macbeth color checker does not provide a significant improvement since these 
24 patches already cover a large part of this low-dimensional spectral space. However, in case of inter-reflection, the apparent spectral reflectances are the results of natural reflectance multiplications, and they are not completely linearly related to the natural reflectances. In the following experiment, this observation is studied in practice.

Let us extract $n$ linear basis functions of the 24-patches Macbeth color checker, $n=$ $1, \ldots, 9$, and check how well the natural reflectances can be reconstructed from these basis functions. We project the natural reflectances on these basis and project them back to the reflectance space. The obtained reflectances $\hat{\mathbf{R}}$ are compared with the original reflectances $\mathbf{R}$ thank to the spectral recovery error $S E=100 \frac{\|\hat{\mathbf{R}}-\mathbf{R}\|}{\|\mathbf{R}\|}$ as recommended by [Q]. Figure 3 displays the evolution of these errors according to the dimension of the linear space $n$, for 3 different reflectance sets. The first set corresponds to the 24 reflectances of the Macbeth Color Checker, the ones used for the basis extraction. The second set corresponds the 1269 Munsell reflectances and the last one to the 24 apparent reflectances provided by the inter-reflections of the Macbeth color patches. For each dimension $n$, we have displayed the average spectral recovery error computed over each set. We can see that the reconstruction error of the apparent reflectances is always higher than the 2 other errors, whatever the dimension used. This means that the apparent spectra can not be reconstructed easily from the linear basis of the Macbeth color chart, so they should bring complementary information in the spectral reconstruction process.

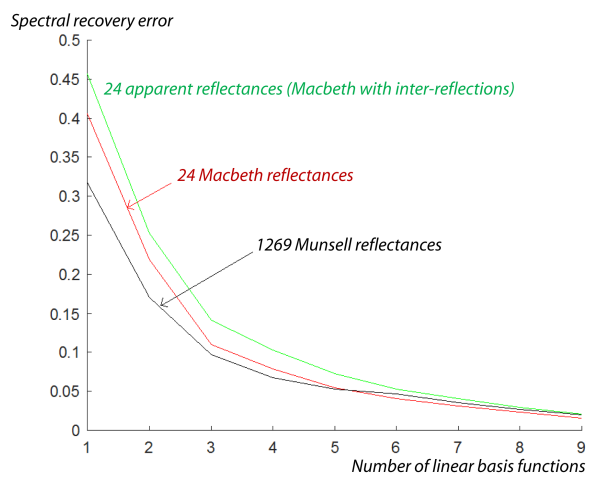

Figure 3: Spectral reflectance reconstruction errors versus dimension.

\section{Experiments}

In this section, we propose to assess the quality of the spectral response reconstruction from raw RGB data, with and without inter-reflections. We first run experiments from synthetic data, as usually done in the litterature $[\mathrm{G}]$, then we reconstruct spectral curves from real color images.

\subsection{Synthetic data}

In this section, we propose to use a physical model in order to construct a synthetic image and then to evaluate spectral sensitivities from these RGB data. For that purpose, we consider 
one known SPD illuminant $\mathbf{E}$ and one known spectral reflectance $\mathbf{R}$ from which we can simulate a spectral radiance vector $\mathbf{L}^{\mathbf{n o}-\mathbf{i n t e r}}$, obtained for a flat surface without inter-reflections thanks to equation (7). This radiance hits the 3 sensors $\mathbf{C}^{\mathbf{R}}, \mathbf{C}^{\mathbf{G}}$ and $\mathbf{C}^{\mathbf{B}}$ of the camera which allows us to get 3 color coordinates $\rho^{R}=\sum_{\lambda} \mathbf{C}_{\lambda}^{\mathbf{R}} \mathbf{L}_{\lambda}^{\mathbf{n o}-\text { inter }}, \rho^{G}=\sum_{\lambda} \mathbf{C}_{\lambda}^{\mathbf{G}} \mathbf{L}_{\lambda}^{\mathbf{n o}-\text { inter }}$ and $\rho^{B}=\sum_{\lambda} \mathbf{C}_{\lambda}^{\mathbf{B}} \mathbf{L}_{\lambda}^{\mathbf{n o}-\text { inter }}$. When creating the synthetic data, we used sensor sensitivities $\mathbf{C}^{\mathbf{R}}$, $\mathbf{C}^{\mathbf{G}}$ and $\mathbf{C}^{\mathbf{B}}$ that are considered unknown at test time. The optimization problem consists in finding the spectral curves $\hat{\mathbf{C}}^{\mathbf{R}}, \hat{\mathbf{C}}^{\mathbf{G}}$ and $\hat{\mathbf{C}}^{\mathbf{B}}$ that minimize the following objective function:

$$
\sum_{i}\left\|\sum_{k \in\{R, G, B\}}\left(k_{i}-\sum_{\lambda} \mathbf{C}_{\lambda}^{\mathbf{k}} \mathbf{L}_{\mathbf{i}_{\lambda}}^{\mathbf{n o}-\mathbf{i n t e r}}\right)\right\|+\alpha\left\|\frac{\sum_{k \in\{R, G, B\}} d^{2}\left(C^{k}\right)}{d \lambda^{2}}\right\| .
$$

The first term is the euclidean distance between the $R G B$ coordinates obtained from the model and the available data. The second term is a regularization one that helps to get smoother sensor sensitivity curves based on the norm of the their second derivative. $\alpha$ enables controlling the balance between the two parts of the equation. $\mathbf{L}_{\mathbf{i}}^{\mathbf{n o}-\mathbf{i n t e r}}$ is the radiance of the $i^{t h}$ patch in the image.

As a second test, we consider folded patches and use equation (9) instead of equation (7) in order to obtain the radiance with inter-reflections. Then we minimize a similar objective function:

$$
\sum_{i}\left\|\sum_{k \in\{R, G, B\}}\left(k_{i}-\sum_{\lambda} \mathbf{C}_{\lambda}^{\mathbf{k}} \mathbf{L}_{\mathbf{i}_{\lambda}}\right)\right\|+\alpha\left\|\frac{\sum_{k \in\{R, G, B\}} d^{2}\left(C^{k}\right)}{d \lambda^{2}}\right\| .
$$

In this case, we have more input data compared to the previous one. Indeed, if we consider the 24 patches of the Macbeth color checker, $i$ goes from 1 to 24 in equation (10) while it goes from 1 to $24 \times n_{f}$ in equation (11), where $n_{f}$ is the number of facets used to sample each patch. Thus, for each color patch, we consider $n_{f}$ different $R G B$ triplets in the case of inter-reflections (folded patches) but only 1 triplet per patch without inter-reflection (flat patches). Practically, we have tested different level of sampling from $6 \times 6$ to $16 \times 16$ and noticed that $10 \times 10$ is a good tradeoff. The results in this paper are evaluated with $n_{f}=10 \times 10=100$ facets.

Since we are working with synthetic data in this section, we propose to add different levels of noise to these data before running the optimization process.

Figure 4 illustrates the evolution of the spectral recovery error between real sensor sensitivities and estimated ones with respect to the noise introduced in the $R G B$ data. We added Gaussian noise whose standard-deviation is increasing along the horizontal axis. By comparing the two cases with and without inter-reflections, we can see that whatever the noise level is, taking into account the inter-reflections with $3 \mathrm{D}$ color charts always improve the accuracy of the results. Indeed, the spectral recovery error is almost twice higher when considering flat classical color chart than when using folded patches. For information we have added a third curve on this plot, which corresponds to the spectral recovery error that would have been obtained if it was possible to create a very big chart with 1269 color patches that correspond to the 1269 Munsell spectral refectances. Off course no color chart exists with such number of patches and furthermore we can see that when the noise level is increasing $\left(>10^{-7}\right)$, the quality of the reconstruction with these 1269 patches is not better than the one obtained with the 24 folded Macbeth patches. For the sake of clarity we have displayed the results for the noise range $\left[0 ; 10^{-5}\right]$, but it interesting to know that with greater noise such as $10^{-2}$, our method get an error of 81.02 while the method without inter-reflection get 90.67. This confirms the outperformance of our approach for greater noise level. 


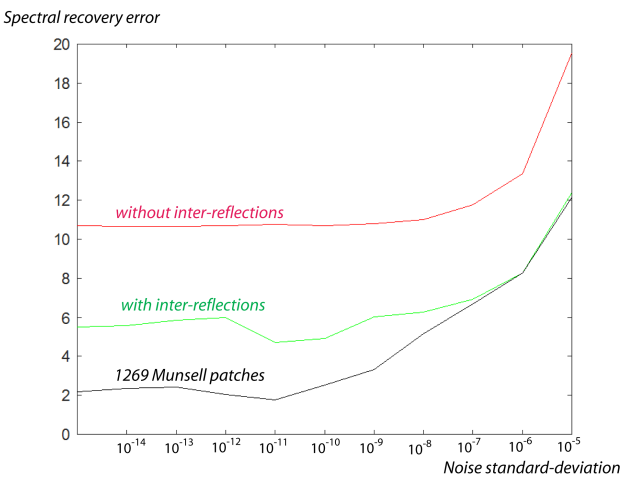

Figure 4: Spectral recovery error versus noise. The horizontal axis represents the evolution of the standard deviation of the added gaussian noise.

Furthermore, we have also tested the sensitivity of the results according to the folded angle. For example, with an error of $10^{\circ}$ in the angle (test with $35^{\circ}$ while using the model for $45^{\circ}$ ), our results still outperform by more than $16 \%$ the ones obtained without interreflections.

\subsection{Real data}

In this section, we acquired real images with the color camera Canon EOS 1000D and reconstruct the spectral sensitivities of its sensors from the obtained raw RGB coordinates. Six different Lambertian surfaces available in our lab were used in these experiments. They are either acquired as planar surfaces or as folded ones depending on the experiments (see figure 5). The spectral reflectances of these patches have been measured in our lab with the Xrite i7 spectro-photometer (one measure per patch, we do not measure the apparent reflectances), as well as the SPD of the light illuminating the scenes during the real acquisitions.
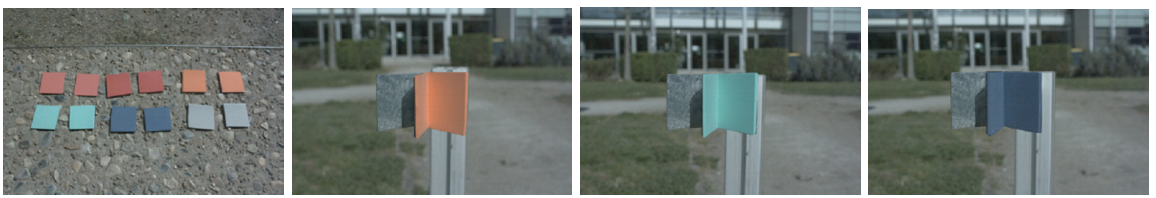

Figure 5: Images of the patches used for the real experiments. Left image: the six patches acquired as planar surfaces, without inter-reflections ( 2 samples per patch). The three right images: three of the six patches acquired as folded surfaces.

We use the same objective functions as the ones presented in the previous section. We reconstruct the sensor sensitivities either from one color image representing the flat patches (left image of figure 5) or from six color images representing the same patches but folded (right images of figure 5). The curves obtained with these two experiments are plotted in Figure 6 . We can see that the spectral reconstructions obtained by taking into account the inter-reflections (in green) are much closer to the ground-truth curves (in red) that the ones 
obtained without these inter-reflections (in black). The associated spectral recovery errors are $13.10 \%$ when inter-reflections are accounted and $23.50 \%$ when they are not.

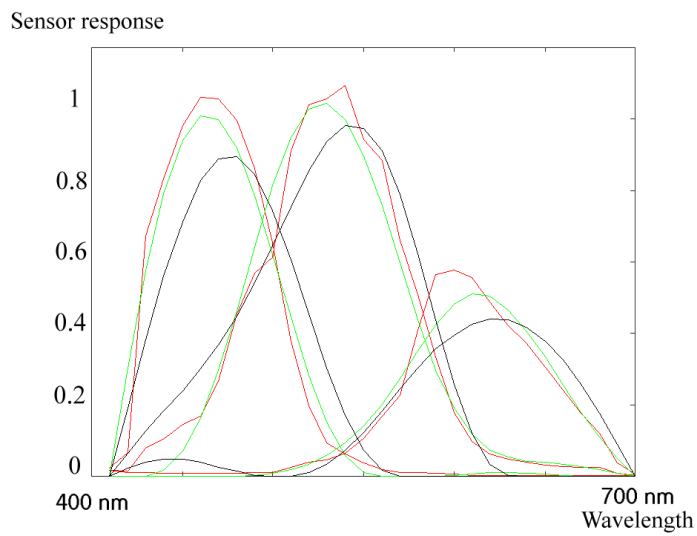

Figure 6: Sensor response reconstructions with and without inter-reflections. Red curve: ground-truth, green curve: reconstruction with inter-reflections, black curve: reconstruction without inter-reflections.

\section{Conclusions and future works}

In this paper, we have studied the advantage of using 3D color charts in spectral camera sensibility estimation. On each folded homogeneous patch, inter-reflections take place introducing different RGB values over its surface. We have demonstrated that these new RGB values are the results of what we called apparent spectral reflectances. These reflectances, compared to the natural reflectances, add less redundancy when used for spectral estimation as they are not completely linearly related to the natural reflectances. Our results showed that using folded patches improves the spectral estimation of sensor sensibility for both synthetic and real data compared to using non-folded patches. One of the future directions is to study inter-reflections when patches with non-homogeneous sides are used. We believe that this case is worth a stand-alone study as the corresponded apparent spectral reflectances are resulted from multiplications of different natural spectral reflectances. This point was studied in [ $[$ ] but, unlike [ $\mathrm{\theta}]$, our solution is based on an infinite-bounces inter-reflection model with sampling points all over the surfaces.

\section{Acknowledgment}

Joost van de Weijer is supported by the project TIN2016-79717-R of the Spanish Ministry of Science. Rada Deeb is visiting the CVC thanks to CMIRA (explora doc) fundings from Region Auvergne-Rhone-Alpes . 


\section{References}

[1] Richard H Byrd, Mary E Hribar, and Jorge Nocedal. An interior point algorithm for large-scale nonlinear programming. SIAM Journal on Optimization, 9(4):877-900, 1999.

[2] T. H. Chan, K. Jia, S. Gao, J. Lu, Z. Zeng, and Y. Ma. Pcanet: A simple deep learning baseline for image classification? IEEE Transactions on Image Processing, 24(12): 5017-5032, 2015.

[3] Maryam Mohammadzadeh Darrodi, Graham Finlayson, Teresa Goodman, and Michal Mackiewicz. Reference data set for camera spectral sensitivity estimation. J. Opt. Soc. Am. A, 32(3):381-391, Mar 2015.

[4] Mark S Drew and Brian V Funt. Calculating surface reflectance using a single-bounce model of mutual reflection. In Computer Vision, 1990. Proceedings, Third International Conference on, pages 394-399. IEEE, 1990.

[5] G.D. Finlayson, S.D. Hordley, and M.S. Drew. Removing shadows from images. In European Conference on Computer Vision (ECCV), pages 823-836, 2002.

[6] Graham Finlayson, Maryam Mohammadzadeh Darrodi, and Michal Mackiewicz. Rank-based camera spectral sensitivity estimation. J. Opt. Soc. Am. A, 33(4):589-599, Apr 2016.

[7] Ying Fu, Antony Lam, Yasuyuki Matsushita, Imari Sato, and Yoichi Sato. Interreflection removal using fluorescence. In European Conference on Computer Vision, pages 203-217. Springer, 2014.

[8] Brian V Funt and Mark S Drew. Color space analysis of mutual illumination. Pattern Analysis and Machine Intelligence, IEEE Transactions on, 15(12):1319-1326, 1993.

[9] Brian V Funt, Mark S Drew, and Jian Ho. Color constancy from mutual reflection. International Journal of Computer Vision, 6(1):5-24, 1991.

[10] Andrew Gilbert and Richard Bowden. Tracking objects across cameras by incrementally learning inter-camera colour calibration and patterns of activity. In 9th European Conference on Computer Vision, pages 125-136. Springer Berlin Heidelberg, 2006. ISBN 978-3-540-33835-2. doi: 10.1007/11744047_10.

[11] K. Helwani, L. Kondrad, and N. Piotto. Low complexity unsupervised multi-camera color calibration with application to panoramic video capturing. In 2015 IEEE International Conference on Image Processing (ICIP), pages 1359-1363, Sept 2015. doi: 10.1109/ICIP.2015.7351022.

[12] Jian Ho, Brian V. Funt, and Mark S. Drew. Separating a color signal into illumination and surface reflectance components: Theory and applications. IEEE Transactions on Pattern Analysis and Machine Intelligence, 12(10):966-977, 1990.

[13] A. Ilie and G. Welch. Ensuring color consistency across multiple cameras. In Tenth IEEE International Conference on Computer Vision (ICCV'05) Volume 1, volume 2, pages 1268-1275 Vol. 2, Oct 2005. doi: 10.1109/ICCV.2005.88. 
[14] Henrik Wann Jensen. Realistic image synthesis using photon mapping, volume 364. Ak Peters Natick, 2001.

[15] Nayar Shree K, Ikeuchi Katsushi, and Kanade Takeo. Shape from interreflections. International Journal of Computer Vision, 6(3):173-195, 1991.

[16] P. Katemake, R. Deeb, D. Muselet, M. Hebert, and A Tremeau. Mutual illumination and color constancy. In 13th AIC (International Color Association) International Congress, October 2017.

[17] R. Khan, J. van de Weijer, D. Karatzas, and D. Muselet. Towards multispectral data acquisition with hand-held devices. In 2013 IEEE International Conference on Image Processing, pages 2053-2057, Sept 2013.

[18] Miao Liao, Xinyu Huang, and Ruigang Yang. Interreflection removal for photometric stereo by using spectrum-dependent albedo. In Computer Vision and Pattern Recognition (CVPR), 2011 IEEE Conference on, pages 689-696. IEEE, 2011.

[19] Jingjing Liu, Shaoting Zhang, Shu Wang, and Dimitris Metaxas. Multispectral deep neural network for pedestrian detection. In British Machine Vision Conference (BMVC), 2016.

[20] David H. Marimont and Brian A. Wandell. Linear models of surface and illuminant spectra. J. Opt. Soc. Am. A, 9(11):1905-1913, Nov 1992. doi: 10.1364/JOSAA.9.001905. URL http://josaa.osa.org/abstract.cfm? URI=josaa-9-11-1905.

[21] Shree K Nayar and Yitao Gong. Colored interreflections and shape recovery. In Image Understanding Workshop, pages 333-343, 1992.

[22] S. W. Oh, M. S. Brown, M. Pollefeys, and S. J. Kim. Do it yourself hyperspectral imaging with everyday digital cameras. In 2016 IEEE Conference on Computer Vision and Pattern Recognition (CVPR), pages 2461-2469, June 2016. doi: 10.1109/CVPR. 2016.270.

[23] Matt Pharr, Wenzel Jakob, and Greg Humphreys. Physically based rendering: From theory to implementation. Morgan Kaufmann, 2016.

[24] Horst Possegger, Thomas Mauthner, and Horst Bischof. In defense of color-based model-free tracking. In The IEEE Conference on Computer Vision and Pattern Recognition (CVPR), June 2015.

[25] Steven M Seitz, Yasuyuki Matsushita, and Kiriakos N Kutulakos. A theory of inverse light transport. In Computer Vision, 2005. ICCV 2005. Tenth IEEE International Conference on, volume 2, pages 1440-1447. IEEE, 2005.

[26] Ralph A. Willoughby. Solutions of ill-posed problems (a. n. tikhonov and v. y. arsenin). SIAM Review, 21(2):266-267, 1979. 